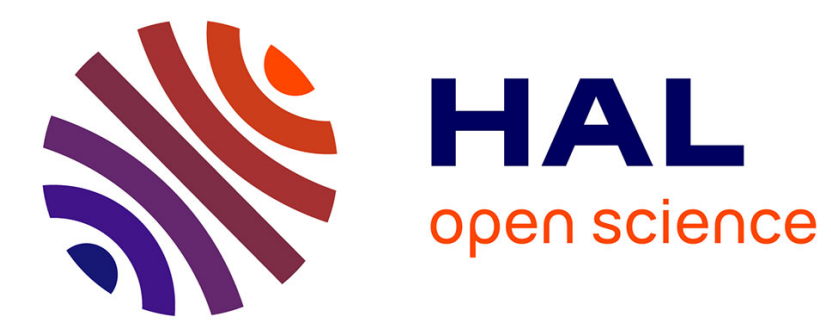

\title{
L'UOIF, la tension clientéliste d'une grande fédération islamique
}

Vincent Geisser

\section{To cite this version:}

Vincent Geisser. L'UOIF, la tension clientéliste d'une grande fédération islamique. Confluences Méditerranée , 2006, 57 (2), pp.83. 10.3917/come.057.0083 • halshs-02113029

\section{HAL Id: halshs-02113029 \\ https://shs.hal.science/halshs-02113029}

Submitted on 30 Apr 2019

HAL is a multi-disciplinary open access archive for the deposit and dissemination of scientific research documents, whether they are published or not. The documents may come from teaching and research institutions in France or abroad, or from public or private research centers.
L'archive ouverte pluridisciplinaire HAL, est destinée au dépôt et à la diffusion de documents scientifiques de niveau recherche, publiés ou non, émanant des établissements d'enseignement et de recherche français ou étrangers, des laboratoires publics ou privés. 


\title{
L'UOIF, LA TENSION CLIENTÉLISTE D'UNE GRANDE FÉDÉRATION ISLAMIQUE \\ Vincent Geisser
}

\author{
L'Harmattan | «Confluences Méditerranée »
}

2006/2 N57 | pages 83 à 101

ISSN 1148-2664

ISBN 2296012663

Article disponible en ligne à l'adresse :

https://www.cairn.info/revue-confluences-mediterranee-2006-2-page-83.htm

Distribution électronique Cairn.info pour L'Harmattan.

(C) L'Harmattan. Tous droits réservés pour tous pays.

La reproduction ou représentation de cet article, notamment par photocopie, n'est autorisée que dans les limites des conditions générales d'utilisation du site ou, le cas échéant, des conditions générales de la licence souscrite par votre établissement. Toute autre reproduction ou représentation, en tout ou partie, sous quelque forme et de quelque manière que ce soit, est interdite sauf accord préalable et écrit de l'éditeur, en dehors des cas prévus par la législation en vigueur en France. Il est précisé que son stockage dans une base de données est également interdit. 


\title{
Vincent GEISSER
}

Chercheur à l'Institut de recherches et d'études sur le monde arabe et musulman (IREMAM-CNRS) et président du Centre d'information et d'étude sur les migrations internationales (CIEMI).

\section{L'UOIF, la tension clientéliste d'une grande fédération islamique}

L'Union des organisations islamiques de France (UOIF), créée en 1983, s'est progressivement imposée comme la principale interlocutrice des pouvoirs publics en matière de culte et de gestion courante des "affaires musulmanes» et elle suscite aujourd'hui beaucoup d'interrogations. Cet article revient sur la dimension "politique» de l'action de l'UOIF, et notamment sur une tendance clientéliste qui lui permet d'apparaître comme un acteur politique à part entiēre : elle produit du sens politique pour soi (exister, être reconnue en tant qu'organisation représentative) et en soi (socialiser, encadrer et mobiliser la "base musulmane»)...

\footnotetext{
"Nous recevons un ami, que nous avons découvert et qui nous a découverts". Thami Breze, président de I'UOIF, à propos de Nicolas Sarkozy, Le Bourget, 19 avril 2003.
}

\begin{abstract}
"C'est vous dire l'importance que j'attache à cette question. J'ai fini par me lier d'amitié avec nombre de mes interlocuteurs musulmans et à me passionner pour la résolution de leurs problèmes". Nicolas Sarkozy, La République, les religions, l'espérance, Paris, Pocket, p. 90.
\end{abstract}

"Protecteurs et protégés sont des amis séparés par l'influence et qui se font des cadeaux selon une certaine

éthique».

Gérard Lenclud, "S'attacher", Terrain, n 21, octobre 1993, p. 14. 


\section{L'UOIF, un objet politique mal identifié}

L'Union des organisations islamiques de France (UOIF), créée en 1983, est sans aucun doute la fédération musulmane de l'hexagone qui suscite aujourd'hui le plus d'interrogations et de passions chez les politiques, les journalistes, les éditorialistes et les chercheurs en sciences sociales. En somme, I'UOIF fascine les esprits français qui sont, tantôt agacés, tantôt admiratifs, mais rarement indifférents à la success story d'une organisation islamique qui s'est progressivement imposée comme la principale interlocutrice des pouvoirs publics en matière de culte et de gestion courante des «affaires musulmanes», éclipsant du même coup le lieu de la «mémoire franco-musulmane» qu'a longtemps incarné la Mosquée de Paris ${ }^{1}$. Pourtant, force est de reconnaître que cette fascination pour I'UOIF - un sigle en quatre lettres produisant un effet quasi-magique sur les «consciences éclairées» - s'est rarement traduite par une investigation approfondie sur les productions et les rouages internes de l'organisation. Mis à part quelques travaux universitaires ${ }^{2}$ et une enquête journalistique rigoureuse $e^{3}$ le «terrain UOIF» reste largement inexploré, telle «une comète islamique», dont le rayonnement attirerait irrésistiblement le regard de l'observateur mais qui, dans le même temps, le dissuaderait de s'en approcher par peur d'être brûlé ou foudroyé. Aussi, malgré l'abondance des discours produits à son propos, I'UOIF est-elle toujours un «objet politique mal identifié» (OPMI), échappant très largement aux analyses sociologiques ou, pour être plus précis, aux tentatives de conceptualisation. Parce qu'elle serait occulte, secrète, insondable, avançant à visage masqué dans I'espace public français, I'UOIF justifierait un «discours d'exception», n'ayant que peu de prise avec les catégories habituellement en usage dans les sciences sociales. D'une manière générale, les «analyses» sur I'UOIF ressortissent du registre du dévoilement : pour ses énonciateurs, il s'agit de mettre en lumière le projet politique des «fréristes français» - l'association étant souvent considérée comme la branche hexagonale des Frères musulmans - qui, sous couvert de culte et de religion, chercheraient, en réalité, à conquérir le pouvoir politique et asseoir un contrôle quasi-totalitaire sur la communauté musulmane de France, évoquant ainsi le spectre de la guerre civile 
en Algérie. C'est notamment la thèse centrale de la politiste Fiametta Venner, dont l'essentiel du propos vise précisément à démasquer le «plan secret» de l'association islamique : «[...] Les dirigeants de l'UOIF ont des objectifs clairs et une stratégie de conquête. Cette organisation a des visées politiques en direction des pays arabomusulmans. Dès sa création, l'UOIF a voulu faire de la France une base arrière: un lieu où des militants islamistes pouvaient trouver refuge et où l'on essaie de modifier le regard que porte l'opinion publique sur leurs pays d'origine. Mais l'UOIF prétend surtout mettre au pas les musulmans de Francen".

Dans le cadre de cet article, nous n'avons pas l'intention de nier la dimension «politique» de l'action de I'UOIF. Au contraire, nous considérerons cette fédération islamique comme un acteur politique à part entière : elle produit du sens politique pour soi (exister, être reconnue en tant qu'organisation représentative) et en soi (socialiser, encadrer et mobiliser la «base musulmane»). Toutefois, la plupart des «analyses» sur I'UOIF nous semblent limitées sur le plan heuristique par le fait qu'elles restent prisonnières de schémas d'interprétation extrêmement normatifs sur ce que devrait être ou ne pas être le «bon islam de France». D'aucuns considèrent, en effet, qu'il faut ostraciser les dirigeants de I'UOIF parce qu'ils seraient dangereux pour la démocratie ( $F$. Venner, $C$. Fourest, L. Babès) $)^{5}$, d'autres qu'il convient, au contraire, de les insérer au «jeu institutionnel» français («intégrer les intégristes») pour mieux les maîtriser (F. Frégosi) $)^{6}$ ou d'autres encore qu'il faut les remplacer par des cadres islamiques plus jeunes et davantage en phase avec la «base musulmane» (Tariq Ramadan, Yamin Makri...).

Prenant nos distances avec ces trois interprétations dominantes, nous tenterons de démontrer que I'UOIF constitue bien un vecteur de politisation de l'islam en France et ceci d'un double point de vue.

L'action de l'UOIF peut être, d'abord, identifiée à celle d'un groupe d'intérêt, voire d'un groupe de pression ${ }^{8}$, qui s'insère dans un espace de représentation islamique compétitif et mouvant. A ce niveau, la dimension «politique» de I'UOIF ressort autant de sa stratégie interne à l'égard de sa «base musulmane» que des interactions produites avec les autres organisations musulmanes (Mosquée de Paris, FNMF...), les médias et les pouvoirs publics : «Un groupe est donc toujours un regroupement, un construit perpétuellement 
retravaillé tant par les modifications de ses 'caractéristiques morphologiques', par les pratiques individuelles et collectives de ses membres et le travail d'énonciation de ses porte-parole prétendants, qui, en le nommant et en le délimitant produisent et mettent en forme des intérêts». En ce sens, on peut avancer l'hypothèse que I'UOIF constitue aujourd'hui l'un des principaux énonciateurs de I'«intérêt musulman» en France, en dépit des critiques qui peuvent surgir ici et là sur sa réelle représentativité.

La dimension politique de I'UOIF ressort ensuite du mode relationnel qu'elle a progressivement instauré avec les autorités publiques locales et nationales qui se décline de plus en plus sur un registre clientéliste. Certes, ce clientélisme peut sembler contradictoire avec la mobilisation des intérêts collectifs musulmans. Ce sont deux «ogiques», deux «registres» que les sociologues opposent fréquemment. Mais, dans le cas de l'UOIF, le clientélisme qui se dégage de son action vient jouer le rôle de modérateur et de réducteur de «tension», engendré par son caractère lobbyiste. Pour être pleinement reconnue par les pouvoirs publics et les institutions, I'UOIF ne peut se contenter d'agir exclusivement sur le mode du rapport de force. Elle est donc contrainte de s'insérer dans un mode relationnel qui relève, en grande partie, du rapport de clientèle, largement encouragé par l'Etat républicain et récemment réactivé, voire amplifié, par l'actuel ministre de l'Intérieur. Comme le rappelle fort pertinemment, Rémy Leveau, «dans la tradition française modelée au cours du XIXe siècle, après la tourmente révolutionnaire, l'Etat désigne comme interlocuteurs ceux qu'il entend voir représenter la communauté. Il leur procure ensuite la reconnaissance et les ressources qui leur permettront de se constituer un réseau clientéliste et de jouer pleinement leur rôle d'intermédiaire» $\aleph^{10}$. De ce point de vue, I'UOIF fait de plus en plus figure de client consentant de la «nouvelle politique musulmane» (NPM) façonnée par Nicolas Sarkozy. A certains égards, elle apparaît même comme le «client musulman idéal» du ministère de l'Intérieur, dans la mesure où elle offre à la fois l'image performative d'une organisation qui dispose d'une «vraie base musulmane» - contrairement à la Mosquée de Paris par exemple - et l'image rassurante d'un «fondamentalisme légaliste et conciliant» contrairement à Tariq Ramadan perçu comme «rebelle» à l'autorité ministérielle ${ }^{11}$ - , ce qui fait d'elle un interlocuteur «crédible» auprès des pouvoirs publics. 
L'UOIF, la tension clientéliste d'une grande fédération islamique

C'est sous ce double registre du groupe d'intérêt musulman et du client consentant que nous allons analyser le rôle politique de I'UOIF dans l'espace public français.

\section{Un groupe d'intérêt musulman : I'UOIF c'est l'islam de France, I'islam de France c'est I'UOIF !}

Lors de l'Assemblée générale du 30 septembre 2005, le président de I'UOIF, Lhaj Thami Breze, définissait ainsi la mission de son organisation : «La réalité de l'UOIF se résume par les éléments suivants : l'UOIF comme référence pour les médias sur les questions relatives à l'islam. L'UOIF comme organisation reconnue auprès de l'opinion publique et auprès de l'opinion musulmane» ${ }^{12}$.

Il énonce clairement ici les lignes d'un programme d'action que les politistes identifient habituellement sous les traits d'un «groupe d'intérêt» ou d'un «groupe de pression», à savoir : un travail permanent de mise en forme symbolique et matérielle de l'sintérêt musulman» à l'échelle nationale et aux échelons locaux (1) ; une consolidation de l'hégémonie organisationnelle par une oligarchie musulmane qui s'érige en porte- parole du «groupe» et s'auto-habilite à dire la «norme islamique» (2), comme l'a très bien montré Khadija Darif dans son étude sociologique sur les cadres dirigeants de I'UOIF.

\section{Matérialisation et symbolisation de "l'intérêt musulman» en [de] France}

Le principal défi pour I'UOIF est de transformer le poids démographique des musulmans de France (nombre de croyants et de pratiquants potentiels) en réalité politique (influence et visibilité) qui fasse pleinement sens dans l'espace public hexagonal. Le travail des dirigeants islamiques va donc consister à accélérer le processus d'islamisation du «groupe latent», en opérant une conversion du groupe musulman en soi (poids démographique) en groupe musulman pour soi (poids politique), à l'instar de ce qu'a pu être le travail «d'ouvriérisation matérielle et symbolique» ${ }^{3}$, mené par les syndicats à l'égard des ouvriers au début du siècle dernier. 
Simultanément, l'organisation doit être capable de se faire reconnaître comme «représentative» du groupe en question, quitte à ce que cette représentativité fasse l'objet d'un débat contradictoire. Mais, d'une certaine manière, la polémique publique, est déjà en soi un signe d'institutionnalisation du groupe d'intérêt. Les dirigeants de I'UOIF ont d'ailleurs bien compris cette logique de «représentativité polémique», répétant souvent l'argument : «Le plus important c'est qu'on parle de nous ! $\rangle^{14}$.

«Exister» implique d'abord de rassembler au sens matériel du terme, c'est-à-dire être capable de se doter de structures d'encadrement, de socialisation et de mobilisation de la «base musulmane», voire d'instaurer ponctuellement un rapport de force avec les pouvoirs publics au nom de la défense de «'intérêt musulman». Cette première dimension - la matérialisation de l'intérêt musulman renvoie à l'épopée organisationnelle de I'UOIF qui se décline sous la forme d'un islamic dream que lui envient aujourd'hui de nombreuses associations et fédérations musulmanes concurrentes. Dans son travail de recherche, $K$. Darif a bien montré comment l'enracinement de I'UOIF sur le territoire français s'attelait à une «réussite matérielle» au sens premier du terme, c'est-à-dire à une structuration territoriale cohérente et performante ${ }^{15}$, à l'image d'une firme commercia$1 e^{16}$ qui aurait pleinement réussi son implantation dans les différentes régions. D'où la nécessité de faire un «détour» par les histoires locales de I'UOIF pour bien comprendre que sa réussite doit moins à une planification centralisée - un plan d'islamisation défini préalablement par une «technocratie islamique» - qu'à une synergie de succès locaux et sectoriels. A ce niveau, I'UOIF constitue bien une «fédération», au sens où les associations locales relativement «autonomes» par rapport au siège national (La Courneuve), ont contribué chacune à leur niveau à asseoir la success story de l'ensemble du mouvement. En somme, l'épopée de I'UOIF se fonde sur une synergie d'épopées locales, résultant de la mise en commun d'expériences islamiques inscrites dans des contextes sociaux et régionaux particuliers.

A Bordeaux, par exemple, ville pionnière de la fédération, la préhistoire de I'UOIF se confond avec l'action en milieu étudiant. Ici, la réalité matérielle de l'organisation islamique, c'est-à- dire la matérialisation du groupe d'intérêt musulman, renvoie à «'ambiance 
universitaire», aux amphis, aux cités étudiantes et aux «resto $U »$, lieux de prédilection, au sein desquels les futurs dirigeants nationaux de l'UOIF feront leurs premières «armes» en matière de militantisme islamique.

Dans le Nord (Lille, Roubaix, Valenciennes..), autre «région phare» de I'UOIF, la matérialisation de l'intérêt collectif «musulman» s'inscrit dans une configuration totalement différente. L'activisme islamique se décline moins sur les registres universitaire et intellectuel que sur les registres populaire (les ouvriers) et ethnique (les immigrés majoritairement originaires du Nord du Maroc). En somme dans la région de Lille, la future élite islamique a accompli ses premières expériences militantes dans une réalité prolétarienne relativement homogène sur le plan ethnique et la concrétisation de son islamic dream a revêtu des accents populaires chtimis (l'islam des corons et des terrils).

Dans d'autres villes françaises, moins importantes en termes d'influence, la matérialisation de l'intérêt musulman, a emprunté les chemins plus modestes de l'association de quartier, de la salle de prière, de l'action de proximité auprès des anciennes et des nouvelles générations de pratiquants musulmans, contribuant malgré tout à la «fabrication» de la future élite islamique à l'échelon local et/ou national.

En définitive, la capacité de l'UOIF à s'imposer progressivement comme la porte-parole du «groupe d'intérêt musulman» dans l'espace public français ne repose pas sur un processus univoque mais sur une forme de synergie des terroirs islamiques locaux, ce qui contredit, en partie, l'idée d'une organisation «totale», voire «totalitaire», porteuse d'un plan de conquête de l'hexagone. Il s'agit moins d'une matérialisation ou d'une structuration unitaire, centralisée et homogène de l'intérêt musulman que d'une mise en réseaux d'expériences locales et régionales. En retour, la direction actuelle est contrainte de respecter une forme de pluralité interne à l'organisation, en accordant une certaine autonomie aux associations affiliées (environ 200), mode de structuration «souple» que l'on retrouve dans la plupart des groupes d'intérêt. A ce niveau, I'UOIF ne fait pas exception : «Plus l'intérêt est complexe (par les agrégations successives qui sont au principe de sa construction), souligne M. Offerlé, plus les intéressés potentiels sont nombreux, plus il sera nécessaire de prévoir ou plutôt de préserver les traces d'une diversité interne produite 
par les processus sociaux...Délimitations stables et délimitations labiles au double sens du résultat momentané des luttes internes et externes de délimitation ${ }^{{ }^{7}}$.

«Exister», c'est aussi accomplir un travail symbolique qui consiste pour I'UOIF à se construire un label de représentativité, «faire labelliser le groupe comme communauté, comme minorité ou comme totalité potentielle» ${ }^{18}$.

Ce processus de labellisation de l'«intérêt musulman» induit, d'abord, chez les dirigeants de I'UOIF une confusion volontaire entre I'histoire particulière de l'organisation et I'histoire collective du groupe «musulmans». L'acte de fondation de I'UOIF (1983) est ainsi identifié par les élites ittihadies ${ }^{19}$ comme l'acte de naissance de la «communauté musulmane de France» dans son ensemble. II s'agit d'une mise en récit rétrospective de I'hégémonie de I'UOIF et de sa légitimité à parler au nom de tous les musulmans de l'hexagone, comme s'il y avait un «avant» et un «après» UOIF dans I'histoire de l'islam de France :

«Le monde associatif musulman est encore petit à cette époque, certains de ses responsables vont naturellement se connaître et s'approcher. C'est ainsi que naît l'Union des Organisations Islamiques en France. Cette naissance va marquer une étape déterminante dans l'évolution de la communauté musulmane vivant en France car elle va augurer la naissance de la communauté musulmane de France». (Discours de Boubakeur El Hadj Amor, 22ème Rencontre annuelle des Musulmans de France, Le Bourget, 25 au 28 mars 2005).

Ce travail symbolique de labellisation suppose, ensuite, une mise en avant de son autonomie organisationnelle à l'égard des autres associations musulmanes (supposées non représentatives), des pouvoirs publics mais aussi des Etats d'origine. L'UOIF véhicule un discours d'autonomie, en se posant comme l'unique défenseur de l'intérêt général musulman de France, par- delà les intérêts particularistes, ethniques et régionalistes:

"L'UOIF réfute toute autre démarche n'émanant pas de ces principes. L'UOIF est une instance indépendante. Elle est guidée dans ses activités, opinions ou attitudes par le seul intérêt de la communauté musulmane de France et le souci d'ordre, d'harmonie et de bien être de la société française» («L'Union se présente», 
http://www.uoif-online.com/uoif.php).

La labellisation implique, enfin, un travail de «nationalisation» de sa rhétorique officielle, de ses références et de son sigle, registres de «francisation» qui ne sont pas simplement «stratégiques» ou «opportunistes» mais aussi «ressentis», apparaissant comme les produits d'un long processus d'autochtonisation de l'organisation et de ses dirigeants :

«En 1987 la charte interne de l'UOIF prend le pas et opère un changement majeur dans les priorités. De 'la sauvegarde' et l'accompagnement des musulmans en séjour en France, celle-ci fixe désormais comme priorité l'accompagnement de la communauté musulmane de France. C'est-à-dire l'UOIF va cesser de considérer les musulmans vivant en France comme des étrangers amenés à la quitter pour les considérer comme des citoyens français ou amenés à le devenir. Trois années d'élaboration et de travail sur le terrain vont participer à donner naissance au concept de musulmans de France. En 1990 les orientations de la charte sont reportées dans le nom. L'Union des Organisations Islamiques en France devient l'Union des Organisations Islamiques de France'». (Discours de Boubakeur El Hadj Amor, 22ème Rencontre annuelle des Musulmans de France, op. cit.).

Mais ce double travail de matérialisation et de symbolisation de la «firme UOIF» n'a pu être pleinement efficace que, parce qu'il a été pris en charge très tôt par des élites islamiques qui, au prix de nombreux compromis et tractations internes, sont parvenues à s'imposer comme les «porte-parole légitimes» de la fédération et, au-delà, de la communauté musulmane de France.

\section{Une "oligarchie islamique» de France : monopolisation de la "parole musulmane» et répres- sion "douce" de la contestation interne}

La consolidation de I'hégémonie de I'UOIF dans le paysage islamique français est consubstantielle d'un «blocage monopolistique de la représentation» par des porte-parole installés. Là aussi, il convient de dépasser les visions simplistes du «complot islamique» téléguidé par une «élite frériste» à la solde d'une organisation étrangère. La confiscation du pouvoir de la «base musulmane» par une élite est 
moins le fruit d'une stratégie pensée et planifiée que le prolongement «ogique» d'une trajectoire organisationnelle complexe, où se dégage progressivement une «oligarchie cultuelle» qui identifie son destin personnel à celui du «collectif musulman». En effet, les dirigeants actuels de I'UOIF vivent leur mandat à la tête de I'organisation non comme une élection politique ou comme une forme de cooptation institutionnelle mais d'abord comme une mission quasiprophétique et divine. Ils se considèrent comme les bâtisseurs de l'islam de France et la moindre menace qui pointe sur l'édifice tend à être interprétée comme une menace collective, mettant en péril I'ensemble de la communauté musulmane française. La consécration des dirigeants à la tête de la fédération procède donc moins d'une manipulation ou d'une «OPA $»^{20}$, que d'une conformation à I'habitus de I'organisation (UOIF) : «accéder à des postes de responsabilité demande du temps et implique une conformation aux savoir-faire, aux manières d'être, à l'habitus d'organisation qui les a élevés. Parce que leurs ressources initiales sont sans commune mesure avec les ressources collectives auxquelles ils accèdent en tant que porte-parole de l'organisation qui les a faits. S'ils tiennent l'organisation c'est

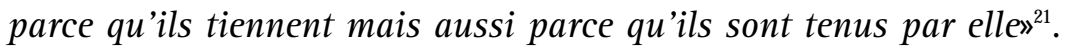

Cette autoreprésentation de leur «mission», largement intériorisée par les simples adhérents et militants de I'UOIF, permet de comprendre le fait qu'aujourd'hui les «négociateurs» et les partisans du compromis soient considérés comme les dirigeants les plus aptes à porter la parole et à défendre les intérêts du mouvement, marginalisant du même coup les élites islamiques critiques. De ce point de vue, la défense de l'intérêt musulman n'est pas incompatible avec le déploiement de logiques clientélistes à l'échelle nationale (ministère de l'Intérieur) et aux échelons locaux (mairies, conseils généraux, conseils régionaux...). Comme le rappelle fort justement le politiste Jean-François Médard, les logiques de mobilisation collective autour d'un intérêt (ici la défense de «'intérêt musulman») peuvent s'accommoder aisément de pratiques clientélistes: «Les exemples ne manquent pas de groupes d'intérêt qui entretiennent des relations privilégiées avec des partis ou des administrations. Ces relations gagneraient à être interprétées dans une optique clientéliste" ${ }^{22}$. C'est précisément ce que nous allons faire en mettant à nu les relations de type «clientéliste» entre I'UOIF et le ministre de I'Intérieur, Nicolas Sarkozy. 


\section{L'UOIF, client consentant de la "nouvelle politique musulmane» de Nicolas Sarkozy}

La question du clientélisme dans les rapports entre l'Etat et les groupes particularistes n'est pas nouvelle en France et constitue même un mode relationnel dominant qui vient relativiser dans les faits le credo républicain, selon lequel les institutions publiques traiteraient avec des «citoyens libres» et non avec des représentants d'intérêts particuliers. Le domaine confessionnel ne fait pas exception, l'Etat républicain étant toujours tenté d'instrumentaliser les groupes religieux. Les observations du politiste Guy Hermet à propos des régimes à «pluralisme limité» pourraient s'appliquer aisément à la démocratie française du XXIe siècle: «Tous les gouvernements souhaitent voir les organisations religieuses remplir des fonctions qui leur soient directement utiles, ou qui soient au moins inof-

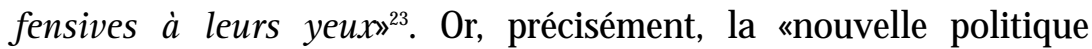
musulmane» initiée par Nicolas Sarkozy nous parait s'inscrire parfaitement dans cette tradition républicaine d'instrumentalisation étatique des organisations religieuses et/ou communautaires, mais en lui donnant un contenu davantage «affectif» et «personnel» qui renforce encore davantage sa dimension clientéliste. A cet égard, on peut dire que le ministre de l'Intérieur n'a rien inventé : il a porté à leur paroxysme des logiques clientélistes en direction des «communautés» (juive, musulmane, chrétiennes...) qui existaient déjà dans le système républicain français. S'il fallait situer philosophiquement son action politique, celle-ci s'inscrirait en filiation moins avec $A$. Tocqueville - que le ministre de l'Intérieur aime pourtant citer ${ }^{24}$ qu'avec les tenants du pluralisme de «ype communautaire» (R.A. Nisbet) pour qui «la finalité essentielle de la démocratie serait d'harmoniser les loyautés de groupe plutôt que de les supprimer ou de les restreindre dans l'intérêt d'un Etat moniste» ${ }^{25}$.

Aussi, les analyses contemporaines sur le clientélisme nous semblent-elles pouvoir décrire, en partie, les relations actuelles entre le ministère de I'Intérieur et I'UOIF, celles-ci relevant d'un clientélisme consenti : «N'étant plus limitée aux rapports entre personnes, on peut appliquer l'analyse clientéliste aux rapports entre groupes ou organisations dans la mesure où la logique des rapports se révèle 
être de nature clientéliste». On peut ainsi envisager une situation de "superposition de clientèles individuelles et collectives»".

\section{Fouad et Nicolas : une gestion affective et personnalisée du dos- sier "/slam»}

Le «tournant clientéliste» de I'UOIF n'est pas concomitant à l'arrivée au ministère de l'Intérieur et des cultes de Nicolas Sarkozy mais s'inscrit dans la longue durée. On peut le faire remonter, d'une part, à 1993, date à laquelle la ligne revendicative de I'UOIF est marginalisée - le Tunisien Abdallah Ben Mansour quitte le secrétariat général - au profit de la ligne conciliatrice, animée par le «clan des exBordelais» - les Marocains Fouad Alaoui et Thami Breze prennent les rênes de la direction -, d'autre part, à 1999, année où l'organisation islamique accepte de participer au processus de consultation (Istichara), initié par J ean-Pierre Chevènement. II s'agit de deux dates clefs pour comprendre l'évolution de I'UOIF qui choisit en quelque sorte d'euphémiser volontairement son rapport de force à l'égard des pouvoirs publics pour privilégier une spolitique de la reconnaissance». Dès lors, les conditions d'une relation de type clientéliste semblent réunies, même si, à l'époque, I'UOIF n'a pas encore trouvé «son patron». Car, précisément, un rapport de clientèle ne se décrète pas mais prend corps dans les modes relationnels concrets, faisant largement appel aux registres affectifs et émotionnels, voire amicaux ${ }^{27}$. En somme, le déploiement de la configuration clientéliste «UOIF-Sarkozy» peut se résumer à I'histoire d'une rencontre «heureuse» entre un acteur communautaire (une fédération islamique), désireux d'obtenir une reconnaissance institutionnelle rapide et un acteur politique pragmatique (un ministre de I'Intérieur), à la recherche d'un interlocuteur musulman «crédible» et relativement indépendant des Etats étrangers. Dès Iors, I'analyse de la relation clientéliste revient à étudier les dispositions des deux partenaires, d'une part, le «patron» (N. Sarkozy) et, d'autre part, le «client» (I'UOIF).

Du côté du ministre de l'Intérieur, également président de I'UMP et candidat déclaré à l'élection présidentielle de 2007, s'exprime dès le départ une volonté très nette de rompre avec la gestion impersonnelle, technocratique et froide du dossier «slam» qui avait marqué la pratique de ses prédécesseurs au ministère des cultes. La 
«proximité» et le «pragmatisme» deviennent désormais les maîtremots de son action cultuelle, légitimant ainsi, auprès de ses interlocuteurs les plus réticents, l'établissement de relations quasi-intimes avec une organisation musulmane réputée pourtant «fondamentaliste»:

«Oui, j’ai voulu qu'il y ait, au sein du CFCM, l'ensemble des représentants $d u$ monde cultuel musulman, y compris l'UOIF. Et je ne l'ai jamais regretté. L'UOIF a toujours respecté sa parole. Les partisans d'un islam plus épicé ont leur place à l'intérieur de cette instance, où ils ont apporté leur représentativité, sans jamais trahir l'esprit de l'instance. Si l'UOIF avait refusé d'y participer ou en était partie, c'est la représentativité du CFCM qui se serait trouvée engagée. Et dans les banlieues, on aurait fait alors de l'UOIF une organisation de martyrs, et du CFCM une coquille $\dot{a}$ moitié pleine. Je ne l'ai pas voulu et j'ai assumé cette responsabilité» (Nicolas Sarkozy, discours au Colloque La laïcité française dans son contexte international : singularité ou modèle?, Académie des sciences morales et politiques, Paris, 19 septembre 2005).

Bien sûr, le clientélisme sarkozien n'est pas exempt de l'affirmation d'un certain paternalisme à l'égard de la «communauté musulmane», en général, et de I'UOIF, en particulier. Tel un «patron politique» vis-à-vis de ses «protégés», N. Sarkozy se présente volontiers comme l'«ami» et I'«avocat» des musulmans de France:

«C'est un paradoxe que je sois devenu 'l'avocat des musulmans' dans la République. La France est capable d'inventer, de faire nầtre des communions étonnantes. C'est parce que je ne suis pas musulman et que mon combat en faveur du rétablissement de l'autorité républicaine était sans ambiguïté que mon devoir était justement d'être aux côtés de ceux qui souffrent au quotidien de l'amalgame et d'une certaine forme de racisme» (Nicolas Sarkozy, «a République, les religions, l'espérance», op. cit., p. 92).

Nous sommes bien en présence ici d'une «amitié paradoxale», telle qu'elle est décrite par le politiste Jean-Louis Briquet à propos du clientélisme politique en $\mathrm{Corse}^{28}$. Mais celle-ci ne se limite pas simplement à une «rhétorique de proximité» sans effets réels. Elle se traduit également par des gestes concrets qui viennent renforcer l'ascendant du «patron Sarkozy» sur son «client musulman», I'UOIF. Outre son déplacement hautement symbolique aux Rencontres du 
Bourget en avril 2003 - le ministre parle d'ailleurs à propos de cet événement de «l'un des grands moments de [sa] vie politique»» N. Sarkozy a su multiplier ces «petits gestes qui font les grands amis» : contacts téléphoniques directs et réguliers avec les dirigeants de I'UOIF - qui ne passent plus désormais par la ligne des «conseillers» -, visites «surprises» au siège de l'organisation islamique à La Courneuve ${ }^{30}$, sans parler des nombreux hommages rendus par le ministre-président à ses «alliés de l'UOIF» dans ses discours publics et ses prestations audiovisuelles. Le président de I'UMP a jeté les bases d'une gestion personnalisée et affective qui constitue l'un des principaux ressorts du clientélisme, lui permettant d'instaurer une relation de confiance avec une organisation qui a longtemps été méfiante à l'égard de l'autorité publique.

\section{Du côté de l'UOIF : la pratique du "compromis permanent» au nom du "juste milieu islamique»}

Toutefois, l'analyse de la relation clientéliste «Sarkozy-UOIF» ne présenterait qu'un faible intérêt heuristique si elle se limitait à un simple rituel politique sans effets de positionnement. Dès lors, la question majeure est : l'établissement d'un rapport de clientèle entre le ministre de l'Intérieur et la fédération islamique a-t-il contribué à infléchir la «igne de l'UOIF» sur un certain nombre de dossiers dits «sensibles»?

Au regard des prises de positions récentes des dirigeants de I'UOIF, nous serions tentés de répondre par l'affirmative. Qu'il s'agisse de l'uaffaire du voile» (avril 2003-mars 2004) ${ }^{31}$, la lutte contre le salafisme ${ }^{32}$, la question palestinienne ${ }^{33}$, le dialogue avec le CRIF (septembre 2004) $)^{34}$, I'uaffaire Tariq Ramadan» (2003-2004)35 ou la gestion sécuritaire de la dernière crise des banlieues (automne $2005)^{36}$, sur tous ces dossiers «sensibles» I'UOIF a adopté une position de «compromis», en produisant un argumentaire de justification théologique, se référant à la nécessité de se conformer au «juste milieu islamique» (el wassatiyya) ${ }^{37}$. Cependant, nous commettrions probablement une erreur, si nous interprétions cette «prudence» de I'UOIF comme la conséquence directe de l'instrumentalisation politique de la fédération islamique par le ministère de I'Intérieur. L'édiction d'une fatwa appelant au retour à l'ordre dans les banlieues, le lâchage de Tariq Ramadan devenu «gênant» - à la suite de 
la publication de son article sur les «nouveaux intellectuels communautaires $\aleph^{38}$ - , I'humanitarisme à propos de la Palestine, la collaboration sécuritaire dans l'éradication des courants salafis, le «dialogue inspiré» avec le CRIF (et non avec le Consistoire ou le Grand Rabbinat) relèvent moins d'une injonction du ministre de l'Intérieur que d'un effet d'anticipation qui prend tout son sens dans la relation de clientèle. II s'agit en quelque sorte de «faire plaisir» à un «patron politique» qui a été le premier à reconnaître la pleine légitimité de I'UOIF dans l'espace public français. Cette notion de «faire plaisir» pourra apparaître anecdotique, voire prêter à sourire, dans le cadre d'une telle analyse sociologique. Elle est pourtant au cœur du dispositif clientéliste «Sarkozy-UOIF» qui tend aujourd'hui à se pérenniser sur un mode relationnel banalisé et qui trouve dans le «juste milieu islamique» un argument d'autorité et une justification théologico-politique auprès des fidèles musulmans.

\section{En guise de conclusion : "Ni ange, ni démon » : la reconnaissance du "Prince» pour horizon d'action}

Nous sommes partis de l'hypothèse que I'UOIF pouvait être considérée comme un acteur politique à part entière, dans la mesure où elle développe une stratégie qui s'apparente à celle d'un groupe d'intérêt, «atténuée», il est vrai, par une insertion de type clientéliste dans les réseaux de pouvoir. Cette mise exergue de son «rôle politique» interdit, toutefois, de penser que la fédération islamique évoluera dans les prochaines années vers une organisation de type «partisan» (un parti des musulmans de France), syndical ou même lobbyiste au sens américain du terme. La thèse du «déclin annoncé de I'UOIF» par excès de notabilisation et coupure avec sa «base musulmane» nous paraît également exagérée. Sur ce plan, nous souscrirons davantage à la thèse de Guy Hermet, relative au positionnement consensuel des groupes religieux qui, selon lui, «s'apparentent d'une certaine manière aux clubs politiques plutôt qu'aux partis. Ils satisfont assez largement les aspirations politiques de ceux de leurs militants qui se sentent frustrés par le monopole idéologique ou 
l'anomie politique que le pouvoir s'efforce d'imposer. Mais ils contribuent en même temps à les stériliser dans la pratique, en enfermant leurs membres dans une sorte de 'cadre d'inaction' propice à une prise de conscience qui ne débouche pas sur une issue concrète, qui devient une fin en soi, et qui ne gêne finalement pas les autorités politiques" ${ }^{39}$.

En définitive, plutôt que de parler de «percée lobbyiste» ou de «déclin irréversible» de l'UOIF, nous préférons conclure en la stabilisation de sa success story islamic autour de trois dimensions majeures qui structureront son action dans l'avenir : le renforcement des pratiques collusives avec les pouvoirs publics français (dialogue et collaboration); I'accentuation des logiques clientélistes à l'échelon national (avec le ministre de l'Intérieur) et aux échelons locaux (avec les maires); et la défense de l'«intérêt musulman» à travers le double registre matériel (les mosquées et les écoles musulmanes) et symbolique (la Palestine, la solidarité islamique internationale...) et sous le contrôle étroit d'une «élite islamique» qui continuera à identifier son destin personnel à celui de l'organisation et, au-delà, à celui de la «communauté musulmane» de France.

Notes :

1. BOYER Alain, L'Institut musulman de la Mosquée de Paris, Paris, CHEAM, 1992.

2. A notre connaissance, le seul travail sociologique sur I'UOIF qui se fonde sur une enquête de terrain est celui de DARIF Khadija, Bricolages identitaires des Musulmans dans l'espace politique français: cas de I'UOIF, mémoire de DEA de science politique, option "Monde arabe et musulman", sous la direction de Vincent Geisser, Institut d'Etudes Politiques d'Aix-en-Provence, 2004.

3. TERNISIEN Xavier, La France des mosquées, Paris, 10/18, 2004. Du même auteur : Les Frères musulmans, Paris, Fayard (coll. "Les dieux dans la Cité»), 2005.

4. VENNER Fiametta, entretien avec Jacqueline Remy et Boris Thiolay, "La face cachée de I'UOIF", L'Express, 2 mai 2005. Cf. aussi son ouvrage : OPA sur l'islam de France. Les ambitions de I'UOIF, Paris, Calman-Lévy, 2005.

5. VENNER Fiametta, OPA sur l'islam de France. Les ambitions de I'UOIF, op. cit. ; FOUREST Caroline, "Les lepénistes de l'islam", Libération, 21 décembre 2004 ; BABES Leïla, "L'UOIF, une minorité radicale», L'Humanité, 25 mars 2005. 


\section{L'UOIF, la tension clientéliste d'une grande fédération islamique}

6. FREGOSI Franck, "Mieux vaut les associer que les écarter», entretien dans L'Express, 2 mai 2005.

7. MAKRI Yamin, "Les 'nouveaux notables' de la République», www.oumma.com, 25 avril 2005. Cf. aussi la déclaration critique de Tariq Ramadan sur I'UOIF citée par TERNISIEN Xavier, Le Monde, 5 avril 2003.

8. C'est aussi l'hypothèse développée par DARIF Khadija, Bricolages identitaires des Musulmans dans l'espace politique français : cas de I'UOIF, op. cit.

9. OFFERLE Michel, Sociologie des groupes d'intérêt, Paris, Montchrestien (coll. "Clefs Politique»), 2e édition, 1998, p. 54.

10. LEVEAU Rémy, "France : changement et continuité de l'islam», dans Rémy Leveau, Khadija Mohsen-Finan, Catherine Wihtol de Wenden (dir.), L'islam en France et en Allemagne, Paris, La Documentation française, 2001, p. 57.

11. Sur la critique sarkozienne de Tariq Ramadan, cf. SARKOZY Nicolas, $L a$ République, les religions, l'espérance, Paris, Pocket, 2005, p. 94-95 (édition originale du Cerf, 2004).

12. BREZE Lhaj Thami, discours à l'assemblée générale de I'UOIF, 30 septembre 2005, http://www.uoif-online.com/uoif.php.

13. OFFERLE Michel, Sociologie des groupes d'intérêt, op. cit., p. 59.

14. C'est un argument que nous avons souvent entendu dans la bouche des dirigeants nationaux et locaux de I'UOIF, lorsque nous les interrogions sur les éventuels effets négatifs (discrédit, ostracisme...) de la stigmatisation médiatique dont ils faisaient l'objet.

15. DARIF Khadija, Bricolages identitaires des Musulmans dans l'espace politique français : cas de I'UOIF, op. cit.

16. HAENNI Patrick, L'islam de marché. L'autre révolution conservatrice, Paris, République des Idées-Seuil, 2005.

17. OFFERLE Michel, Sociologie des groupes d'intérêt, op. cit. p.68.

18. OFFERLE Michel, Sociologie des groupes d'intérêt, op. cit., p. 66-67.

19. Ittihad est la traduction arabe d'Union. Les membres de I'UOIF sont parfois qualifiés d' «ittihadistes» (unionistes).

20. VENNER Fiametta, OPA sur l'islam de France. Les ambitions de I'UOIF, op. cit.

21. Ibid., p. 85.

22. MEDARD Jean-François, "Le rapport de clientèle : du phénomène social à l'analyse politique", Revue française de science politique, année 1976, vol. 
26, $n^{\circ} 1$, p. 126.

23. HERMET Guy, "Les fonctions politiques des organisations religieuses dans les régimes à pluralisme limité», Revue française de science politique, $n^{\circ}$ 3, vol. 23, année 1973, p. 446.

24. Cf. la citation de Tocqueville au début de son ouvrage La République, les religions, l'espérance, op. cit., p. 7.

25. GRAZIANO Luigi, "Le pluralisme. Une analyse conceptuelle et comparativen, Revue française de science politique, n² 2, vol. 46, 1996, p. 198-199.

26. MEDARD Jean-François, "Le rapport de clientèle : du phénomène social à l'analyse politique», op. cit., p. 121.

27. Sur les registres affectifs du clientélisme, cf. BRIQUET Jean-Louis, "Des amitiés paradoxales : échanges intéressés et morale du désintéressement dans les relations de clientèle», Politix, $n^{\circ} 45,1999$, p. 7-19 ; LENCLUD Gérard, "S'attacher", Terrain n 21 - Liens et pouvoir -, octobre 1993, http://terrain.revues.org/document3074.html.

28. BRIQUET Jean-Louis, "Des amitiés paradoxales...», ibid.

29. "Religions, République, intégration. Sarkozy s'explique», entretien avec Denis Jeambar, L'Express, 1er septembre 2004.

30. A notre connaissance, sa dernière visite au siège de I'UOIF remonte au Conseil d'administration de la fédération, le 25 juin 2005.

31. L'UOIF a appelé ses adhérents et ses sympathisants à ne pas prendre part aux manifestations contre la loi sur les signes religieux. Elle a même exercé une forme de répression sur certains de ses dirigeants qui souhaitaient y participer.

32. Dans son ouvrage, N SARKOZY a d'ailleurs remercié I'UOIF pour sa collaboration précieuse dans la lutte contre le salafisme : "La réalité, c'est que I'UOIF mène, sur le terrain, un travail utile contre des adversaires autrement plus dangereux pour la République : les salafistesi, in La République, les religions, l'espérance, op. cit., p. 100.

33. La fédération islamique tout en rendant hommage aux «martyrs palestiniens" évite d'appeler à de grandes manifestations ou rassemblements publics des musulmans de France en soutien à la "cause palestinienne». Elle se cantonne au registre humanitaire. Cf. le discours de Lhaj Thami Breze, assemblée générale de I'UOIF, 30 septembre 2005.

34. Le 9 septembre 2004, une délégation de trois membres de I'UOIF (dont Fouad Alaoui) a rencontré une vingtaine de membres du Conseil représentatif des institutions juives de France.

35. La direction de I'UOIF a dissuadé ses organisations de jeunesse, notamment Etudiants musulmans de France (EMF) et Jeunes musulmans de France (JMF) d'inviter le prédicateur musulman pourtant populaire dans les 


\section{L'UOIF, la tension clientéliste d'une grande fédération islamique}

nouvelles générations.

36. Lors de la "crise des banlieues" d'automne 2005, I'UOIF a publié un communiqué et édicté une fatwa pour appeler à l'arrêt des violences urbaines et au retour au calme des "jeunes musulmans". Cf. le communiqué de I'UOIF, 6 novembre 2005 et la "Fatwa concernant les troubles qui touchent la France», 6 novembre 2005, http://www.uoif-online.com.

37. Sur la notion de "juste milieu", cf. I'analyse pertinente de DARIF Khadija, Bricolages identitaires des Musulmans dans l'espace politique français : cas de I'UOIF, op. cit.

38. Tariq Ramadan, "Critique des (nouveaux) intellectuels communautaires", www.oumma.com, 3 octobre 2003.

39. HERMET Guy, "Les fonctions politiques des organisations religieuses dans les régimes à pluralisme limité», op. cit., p. 470. 